(C) 1999 International Press

Adv. Theor. Math. Phys. 3 (1999) 209-215

\title{
The asymptotics of an amplitude for the 4-simplex
}

\author{
John W. Barrett ${ }^{a}$ and Ruth M. Williams ${ }^{b}$ \\ ${ }^{a}$ Center for Gravitational Physics and Geometry, \\ Department of Physics, \\ The Pennsylvania State University, \\ 104 Davey Laboratory, \\ University Park, PA 16802-6300, \\ USA \\ jwb@maths.nott.ac.uk \\ ${ }^{b}$ DAMTP, \\ Silver Street, \\ Cambridge CB3 9EW, UK \\ R.M.Williams@damtp.cam.ac.uk
}

\begin{abstract}
An expression for the oscillatory part of an asymptotic formula for the relativistic spin network amplitude for a 4 -simplex is given. The amplitude depends on specified areas for each two-dimensional face in the 4-simplex. The asymptotic formula has a contribution from each flat Euclidean metric on the 4-simplex which agrees with the given areas. The oscillatory part of each contribution is determined by the Regge calculus Einstein action for that geometry.
\end{abstract}

e-print archive: http://xxx.lanl.gov/abs/gr-qc/9809032 


\section{Introduction}

The purpose of this paper is to give a physical interpretation of the function of 10 balanced representations of the Lie group $\mathrm{SO}(4)$ introduced in [1]. This value of the function is a real number calculated from a relativistic spin network associated to a 4 -simplex. We call it a symbol, by analogy with the terminology of $6 \mathrm{j}$-symbols for $\mathrm{SU}(2)^{1}$. Each balanced representation is determined by a non-negative half-integer $j$, the spin. The 10 balanced representations are associated to the 10 triangles of the 4 -simplex.The spins are restricted by the requirement that the sum of the spins on the faces of each tetrahedron is an integer.

'In this paper we determine the leading part of an asymptotic formula for the symbol, inspired by the corresponding formula for a $6 j$-symbol given by [5]. The formula has a contribution from each metric on the 4-simplex for which the area of the triangle is given by $2 j+1$, where $j$ is the spin label for that triangle. The phase factor for each contribution is determined by the Regge calculus formula for the Einstein action of the 4-simplex. An argument connecting the closely related balanced $15 j$-symbol and the Einstein action was given by [6].

The general context for the symbol as an amplitude in a state sum model was introduced in [1], and developed in [4]. Some more background is explained in the Penn State lecture [2].

\section{The symbol}

The symbol was originally defined in terms of a relativistic spin network evaluation in [1]. For the classical Lie group considered here, this is defined by taking the value of the $15 \mathrm{j}$-symbol for $\mathrm{SU}(2)$ which is associated to the 4 -simplex with 5 additional spins specified at tetrahedra. The square of this number is then summed over the 5 additional spins, with appropriate weights.

The relativistic spin network evaluation was shown to be given by an integral over copies of the Lie group SU(2) in [3]. (In that paper, the integer $n=2 j$ was called the spin.) This definition is used as the starting point for this paper.

\footnotetext{
${ }^{1}$ It is tempting to call this a $10 \mathrm{j}$-symbol, but the terminology $\mathrm{Nj}$-symbol already has a specific meaning, and the symbol is not one of these.
} 
The five tetrahedra in the 4 -simplex are numbered by $k=1,2, \ldots 5$, and the triangles are indexed by the pair $k, l$ of tetrahedra which intersect on the triangle. The 10 spins are thus $\left\{j_{k l} \mid k<l\right\}$.

The matrix representing an element $g \in \mathrm{SU}(2)$ in the irreducible representation of spin $j_{k l}$ belonging to a triangle is denoted $\rho_{k l}(g)$.

A variable $h_{k} \in \mathrm{SU}(2)$ is assigned to each tetrahedron $k$. The invariant $I \in \mathbb{R}$ is defined by integrating a function of these variables over each copy of $\mathrm{SU}(2)$.

The evaluation of the relativistic spin network is

$$
I=(-1)^{\sum_{k<l} 2 j_{k l}} \int_{h \in S U(2)^{5}} \prod_{k<l} \operatorname{Tr} \rho_{k l}\left(h_{k} h_{l}^{-1}\right) .
$$

The integration measure is the Haar measure on each of the five copies of $\mathrm{SU}(2)$, normalised to total volume 1 .

The geometrical interpretation of this formula given in [3] is that since the manifold $\mathrm{SU}(2)$ is isomorphic to $S^{3}$ by

$$
x \mapsto\left(\begin{array}{cc}
x_{0}+i x_{1} & x_{2}+i x_{3} \\
-x_{2}+i x_{3} & x_{0}-i x_{1}
\end{array}\right) .
$$

each variable $h \in \mathrm{SU}(2)$ can be regarded as a unit vector in $\mathbb{R}^{4}$. Further, this unit vector can be regarded as the normal vector to a 3-dimensional hyperplane in $\mathbb{R}^{4}$ through the origin. The idea is to interpret this as the hyperplane in which the tetrahedron lies in a geometrical simplex in $\mathbb{R}^{4}$. Similar variables were introduced in a first order version of classical Regge calculus by [7].

The weight for one triangle $k l$ is a function of the angle $\phi$ between the two unit vectors $h_{k}$ and $h_{l}$ in $\mathbb{R}^{4}$,

$$
\operatorname{Tr} \rho\left(h_{k} h_{l}^{-1}\right)=\frac{\sin (2 j+1) \phi}{\sin \phi} .
$$

This angle, defined by $\cos \phi=h_{k} \cdot h_{l}$, is the exterior angle between the two hyperplanes. Since the sum of the spins at a tetrahedron is an integer, the integrand is unchanged if $h$ is replaced by $-h$ at one tetrahedron. Thus the integrand does not register the orientation of the hyperplanes.

The set of five hyperplanes determines a geometric 4 -simplex in $\mathbb{R}^{4}$ up to overall scale and parallel translation, assuming the generic case where any 
four of the unit vectors are linearly independent. Moving one of the hyperplanes parallel to itself away from the origin, defines a geometric 4-simplex as the space bounded by the hyperplanes. The distance the hyperplane is moved from the origin is undetermined, so the overall scale of the simplex is undetermined. This possible scaling includes negative scaling factors, which invert the 4-simplex.

In this way, the integration can be regarded as an integration over the set of geometric 4-simplexes modulo isometries and scaling. The isometries are products of translations and transformations by elements of $\mathrm{O}(4)$. This set of 4-simplexes is parameterised by the ten angles $\phi$, which are subject to one constraint equation. This is the same as the space of edge lengths for the simplex, modulo scaling all 10 lengths simultaneously.

Note that the scaling by a factor -1 does not change the orientation, so the geometric simplexes up to scaling are oriented simplexes. However the value of the integrand does not depend on this orientation.

\section{Asymptotics}

For large values of the spins, the asymptotic value of the integral can be calculated using the method of stationary phase. The important terms are the $\sin (2 j+1) \phi$, as these depend on the asymptotic parameters, the spins $j$. These are expanded as exponentials to apply the stationary phase method,

$$
\sin (2 j+1) \phi=\frac{1}{2 i}(\exp i(2 j+1) \phi-\exp -i(2 j+1) \phi) .
$$

The $\sin \phi$ factors in the denominator do not vary as $j \rightarrow \infty$, so can be treated as part of the integration measure.

For each triangle, introduce a variable $\epsilon_{k l}$ which can take the values \pm 1 . Then the integral expression for $I$ can be written

$$
I=\frac{(-1)^{\sum_{k<l} 2 j_{k l}}}{(2 i)^{10}} \sum_{\substack{\epsilon_{12}= \pm 1 \\ \epsilon_{45}= \pm 1}} \int_{h \in S U(2)^{5}}\left(\prod_{k<l} \frac{\epsilon_{k l}}{\sin \phi_{k l}}\right) \exp \left(i \sum_{k<l} \epsilon_{k l}\left(2 j_{k l}+1\right) \phi_{k l}\right) \text {. }
$$

Now each term in this sum depends on slightly more than the angles of a simplex. This is because the term is not invariant under the replacement $h \rightarrow-h$. The geometric simplex determined by the hyperplanes has outward normal vectors $n_{k}$ for the tetrahedra, and $n_{k}= \pm h_{k}$ for each $k$. This means that either $\phi$ or $\pi-\phi$ are the exterior angles of the geometric 4-simplex. 
Each integral can be split into a number of domains where these five signs take particular values. Now changing $h \rightarrow-h$ shows that each domain is equivalent to a domain where $n_{k}=h_{k}$ for all $k$, but for different values of the $\epsilon_{k l}$. Therefore we can assume that $n_{k}=h_{k}$, and so the $\phi$ are exactly the exterior angles of the geometric simplex. Now it is possible to compute the stationary points. These are the stationary points of the action

$$
S=\sum_{k<l} \epsilon_{k l}\left(2 j_{k l}+1\right) \phi_{k l} .
$$

The one constraint between the $\phi$ can be taken account of using Schläfli's differential identity [Ponzano and Regge 1968]

$$
\sum_{k<l} A_{k l} \mathrm{~d} \phi_{k l}=0
$$

where the $A_{k l}$ are the areas of the triangles of one of the geometric 4simplexes determined by the $\phi$ (i.e., fixing a particular scale for the 4simplex). This constrained variation is exactly as for the corresponding first order formalism for Regge calculus [8]. Using a Lagrange multiplier $\mu$ for this constraint,

$$
\mathrm{d} S=\sum_{k<l} \epsilon_{k l}\left(2 j_{k l}+1\right) \mathrm{d} \phi_{k l}=\mu \sum A_{k l} \mathrm{~d} \phi_{k l},
$$

and so for each triangle

$$
\epsilon_{k l}\left(2 j_{k l}+1\right)=\mu A_{k l} .
$$

Since the $\left\{A_{k l}\right\}$ are only determined up to overall scale, it is most convenient to take $\mu= \pm 1$ to fix this overall scale. Then the result is that for a stationary phase point in the integrand

1. The $\epsilon_{k l}$ are either all positive or all negative.

2. The angles $\phi$ are those of a geometric 4-simplex with areas $A_{k l}=$ $2 j_{k l}+1$.

3. The integrand is $\exp i \mu S_{E}$, with $S_{E}=\sum_{k<l} A_{k l} \phi_{k l}$ which is the Regge calculus version of the Einstein action for a 4 -simplex, and $\mu= \pm 1$.

The last point about the Einstein action requires a little more explanation. The Einstein action for a 4-manifold is the integral of the scalar curvature on the manifold plus the integral of the mean curvature of the boundary. Here, the scalar curvature is zero and so the action for the flat 4 -simplex is entirely the boundary term [9]. 
Each stationary phase point occurs $2^{5}$ times, so the asymptotic formula is

$$
I \sim-\frac{(-1)^{\sum 2 j}}{2^{4}}\left(\sum_{\sigma} P(\sigma) \cos \left(S_{E}(\sigma)+\kappa \frac{\pi}{4}\right)\right)+D
$$

The sum is over the set of metric 4-simplexes $\sigma \subset \mathbb{R}^{4}$ modulo isometries, which are such that the area of the triangle $k l$ is given by $2 j_{k l}+1$. As the squares of the areas are quadratic polynomials in the squares of the edge lengths, and the mapping is non-degenerate, at least in the regular cases [8], this appears to be a finite set.

The prefactor $P$ is calculated in the usual way for stationary phase in terms of a determinant. This does not oscillate with the asymptotic parameters $j$ and so is quite different to the cosine term. Finally, there is also a contribution $D$ to the asymptotics of the integral from degenerate simplexes, when two of the $h$ 's coincide. We have not analysed these terms. 


\section{References}

[1] J.W. Barrett, L. Crane, Relativistic spin networks and quantum gravity gr-qc/9709028 J. Math. Phys. 39 (1998), 3296-3302.

[2] J.W. Barrett, State sum models for quantum gravity. Penn State relativity seminar August 27, 1998. http://vishnu.nirvana.phys.psu.edu/relativity_seminars.html for audio and text of transparencies. (1998b).

[3] J.W. Barrett, The classical evaluation of relativistic spin networks, math.QA/9803063, Adv. Theor. Math. Phys. 2 (1998a), 593-600.

[4] J.C. Baez, Spin foam models, Class. Quant. Grav. 15(1998), 1827-1858.

[5] G. Ponzano and T. Regge, Semiclassical limit of Racah coefficients, Spectroscopic and group theoretical methods in Physics (F. Bloch, ed.), North-Holland, 1968, pp. 1-58.

[6] L. Crane and D.N. Yetter, On the classical limit of the balanced state sum, gr-qc/9712087, (1997).

[7] M. Caselle, A. D'Adda and L. Magnea, Regge calculus as a local theory of the Poincaré group, Phys. Lett. B232 (1989), 457-461.

[8] J.W. Barrett, First order Regge calculus, Class. Quant. Grav. 11 (1994), $2723-2730$.

[9] J.B. Hartle and R. Sorkin Boundary terms in the action for the Regge calculus, Gen. Rel. Grav. 13 (1981), 541-549. 